\section{Analyses de thèses}

Orthop Traumatol (1991) $1: 188$

\section{H. Gagnière \\ Le phénomène de rétraction longitudinale dans l'utilisation du matelas coquille.}

Thèse Strasbourg, 1992, N 13.

Les premiers modèles d'ambulance furent imaginés sous l'Empire par D. Larrey. Mais ce fut après la dernière guerre mondiale que les systèmes d'urgence furent organisés et à partir de 1960 que les S. A. M. U. firent leur apparition, notamment afin de transporter les blessés de la route.

$\mathrm{Si}$ au départ il s'agit d'acheminer le blessé le plus vite possible par n'importe quel moyen, à partir de 1960 on s'intéresse plus aux "moyens" afin de ne pas aggraver les lésions préexistantes.

C'est un physicien strasbourgeois J. Loeb qui met au point en 1959 un matelas coquille (dont la dépression permet d'épouser les contours du sujet contenu) permettant une immobilisation globale et un maniement

\section{E. Favreul}

Le clou gamma : nouvelle technique d'ostéosynthèse pour les fractures trochantériennes. Résultats à propos de 120 cas.

Thèse Strasbourg, 1991, N ${ }^{\circ} 12$.

En complément des traitements classiques par enclouage de Ender et vis plaque D. H. S. des fractures trochantériennes de l'adulte, le Centre de Traumatologie de Strasbourg a développé un clou centro-médullaire verrouillé à sa partie inférieure et perforé en haut d'une longue vis cervicale. Inspiré du clou en Y de Kuntscher, celui-ci s'adresse en premier lieu aux fractures instables (3 à 8 de la classification de Ender) pour lesquelles l'enclouage de Ender n'assure pas une réduction ni une stabilité suffisante. plus facile. Mais la compression du matelas s'effectue dans tous les axes et est néfaste dans le sens longitudinal risquant une aggravation des lésions traumatiques du rachis et des lésions médullaires. L'auteur s'attache à mettre en évidence la rétraction par des études expérimentales. Les forces de compression varient de 5 à $50 \mathrm{~kg}$ selon que le patient est totalement ou simplement immobilisé latéralement (gouttière).

Une étude tomodensitométrique sur pièces vertébrales fraîches de cadavre avec fracture corporéale a permis de montrer une variation de longueur du rachis de $0,5 \%$ à $2,5 \%$ et de $3,5 \%$ à $33 \%$ du segment fracturé !

Ces chiffres permettent de déduire que le patient ne doit pas être enveloppé complètement. D'autre part un nouveau type de matelas anti-rétraction (en cours d'expérimentation) a été proposé par son inventeur. Les étude semblent montrer une amélioration avec diminution de la compression axiale.

Alain G. Graftiaux

L'étude de 120 cas - comprenant $74 \%$ de fractures instables à 3 ou 4 fragments de la classification de Evans - confirme que le foyer fermé permet de diminuer les pertes sanguines ainsi que la durée opératoire. Ce travail met en relief la possibilité de mettre en charge complète $87 \%$ des patients avant la fin de la première semaine (ce qui est plus précoce qu'avec une vis plaque D. H. S. ). Le cal osseux obtenu est de bonne qualité et il n'y a pas eu de geste complémentaires nécessaire comme c'est souvent le cas avec la vis plaque D. H. S. . La réduction est de bonne qualité et il existe moins de cal vicieux qu'avec l'enclouage de Ender. Les complications observées sont essentiellement mécaniques ( 2 fractures diaphysaires et 6 déplacements secondaires). Mais à 3 mois de recul $73 \%$ des patients ont retrouvé un état fonctionnel antérieur, ce qui l'indique dans tous les types de fractures trochantérienne, à tout âge.

Alain G. Graftiaux 